\title{
Cygnus X-3's "Little" Friend
}

\section{Michael McCollough*}

CXC/SAO

E-mail: mmccollough@head.cfa.harvard.edu

Cygnus X-3 is a well know microquasar which shows state changes, strong radio emission, hard $\mathrm{X}$-ray/X-ray/radio correlations, and relativistic jets. The exact nature of the compact object associated with this system is still uncertain. In this presentation are shown new results which will further illustrate the complexity and uniqueness of this system.

In 2003, using Chandra observations, extended X-ray emission was discovered associated with Cygnus X-3 [3]. The most promising interpretation of this feature was that it is a jet impact region. In 2006 a longer Chandra observation during which Cygnus X-3 was in a brighter X-ray state has revealed a much better look at this emission. Even though this feature is $16^{\prime \prime}$ away from Cygnus X-3 it demonstrates remarkable time correlated activity associated with Cygnus X-3. The behavior of this emission and the possible nature of this feature will be discussed.

VII Microquasar Workshop: Microquasars and Beyond September 1 - 5, 2008

Foca, Izmir, Turkey

\footnotetext{
* Speaker.
} 


\section{Introduction}

Cygnus X-3 is an unusual X-ray binary containing a compact object and a Wolf-Rayet companion, making it a high mass system. But its orbital period $(4.8 \mathrm{hrs})$ is typical for a low mass system. It is a strong radio source routinely producing radio flares of over a Jy and up to $\sim 20 \mathrm{Jy}$ on occasion. Even during radio quiescence it can be relatively bright in the radio (60-100 $\mathrm{mJy}$ ). It has been shown to produce radio jets and also demonstrates correlations of the radio with both the X-ray and hard X-ray [1], 2] .

Using Chandra observations, taken in 2000, extended X-ray emission was discovered associated with Cygnus X-3 [3] . Their analysis pointed to this feature as being a jet impact region. In 2006 a longer Chandra observation during which Cygnus X-3 was in a brighter X-ray state has revealed a much better look at this emission. We present the initial results of an analysis of this unique feature.

\section{Observations}

In early 2006 Cygnus X-3 entered into a radio/hard X-ray quenched state (X-ray: high/soft state) after nearly four years in a radio quiescent state. During this quenched state a $50 \mathrm{ksec}$ Chandra observations (OBSID: 6601) was performed as part of an international observing campaign to study major flaring activity in Cygnus X-3. The Chandra observations were grating observations designed to study the strong stellar winds that are associated with Cygnus X-3.

An image of the observation (zero order of the grading observation) is shown in Fig. 1. The feature lies about $45^{\circ}$ from the orientation of the radio jets that have been observed in Cygnus X3. The direction of the radio jets corresponds roughly to the direction of the instrumental readout streak. The feature is $\sim 16^{\prime \prime}$ from Cygnus X-3. It is extended with a radial size (from Cygnus X-3) of $\sim 3^{\prime \prime}$ and a tangential size of $\sim 5^{\prime \prime}$. If the feature and Cygnus X-3 are at the same distance then their separation is $2.7 \mathrm{D}_{10}$ light-years $\left(\mathrm{D}_{10}\right.$ : distance in units of $\left.10 \mathrm{kpc}\right)$. The feature is a factor of $10^{-4}$ times fainter than Cygnus X-3. As can be seen in Fig. 2 this feature has existed throughout the Chandra mission (1999 to present).

\section{Lack of Counterparts at Other Wavelengths}

Observations at other wavelengths have yet to reveal a counterpart. A recently reported radio feature near Cygnus X-3 [ [] is on the opposite side of Cygnus X-3. An examination of a HST/NICMOS observations taken at 1.76 microns (see Fig. 3) shows no obvious counterpart.

In December 2007 Spitzer observed the region containing Cygnus X-3 with MIPS at 24 and 70 microns with nominal exposures times of a couple of minutes. There was no detection at $70 \mathrm{mi}-$ crons. But Cygnus X-3 is clearly detected at 24 microns. Fig. 3 shows the Cygnus X-3 region and a closeup of Cygnus X-3 at 24 microns. There is some evidence of extended emission associated with Cygnus X-3 and possibly with the feature. But this is a complex area and further analysis will be necessary. 


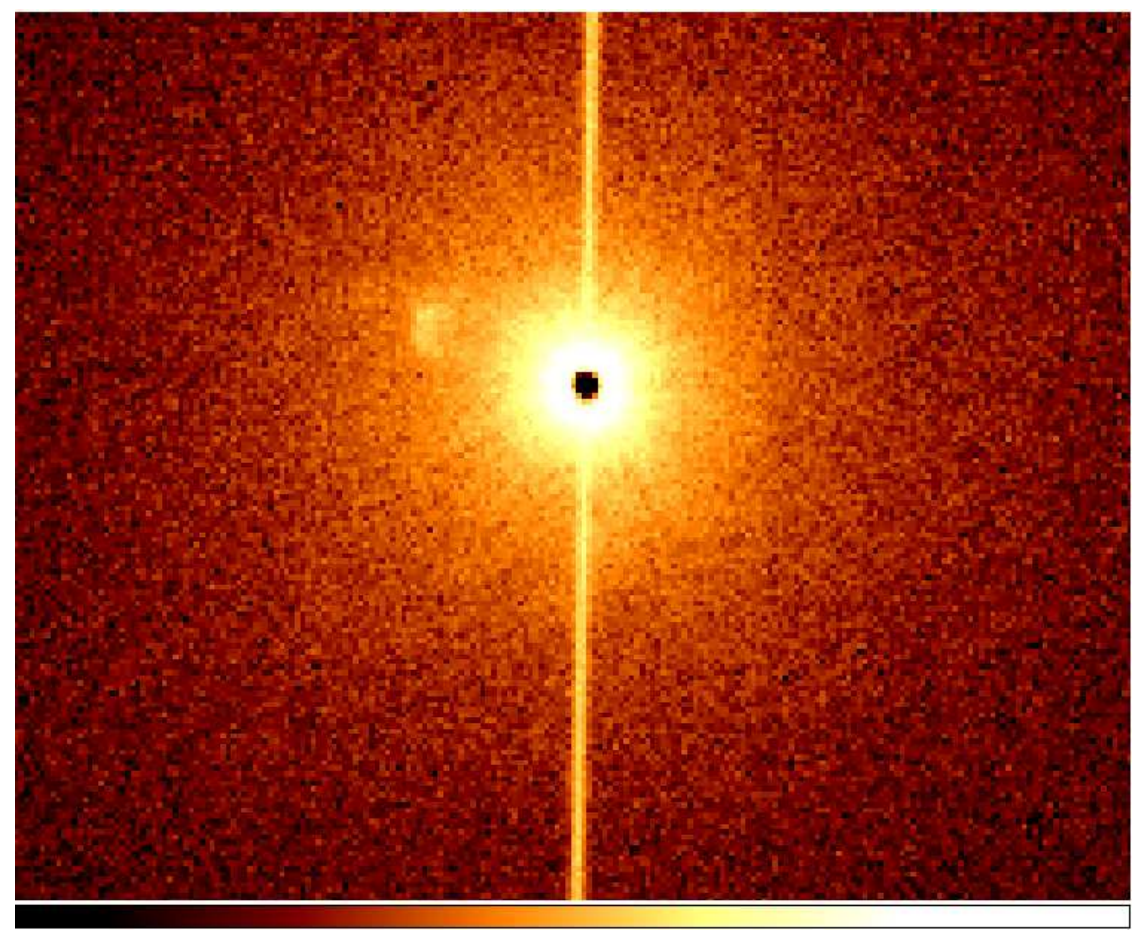

Figure 1: A zero order Chandra grating image of Cygnus X-3. In addition to the feature note two instrumental effects: (a) bright readout streak caused by the ACIS CCD readout; and (b) the "cratering" of the central source due to pileup.

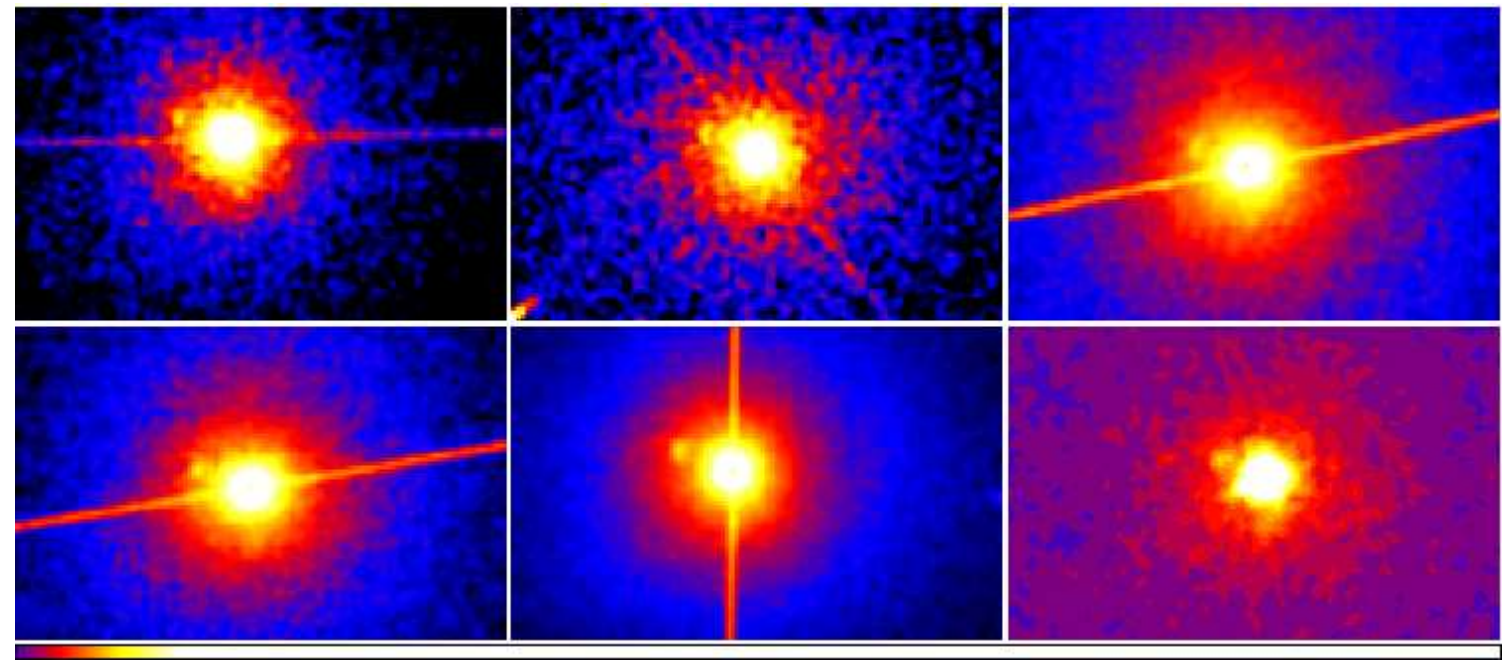

Figure 2: Chandra observations spanning 1999-2006. Five ACIS (HETG) and one HRC (lower right) observations of Cygnus X-3. Note the different spacecraft rolls (orientation of the readout streak) of the ACIS observations. Observations also cover different states of Cygnus X-3's activity. 

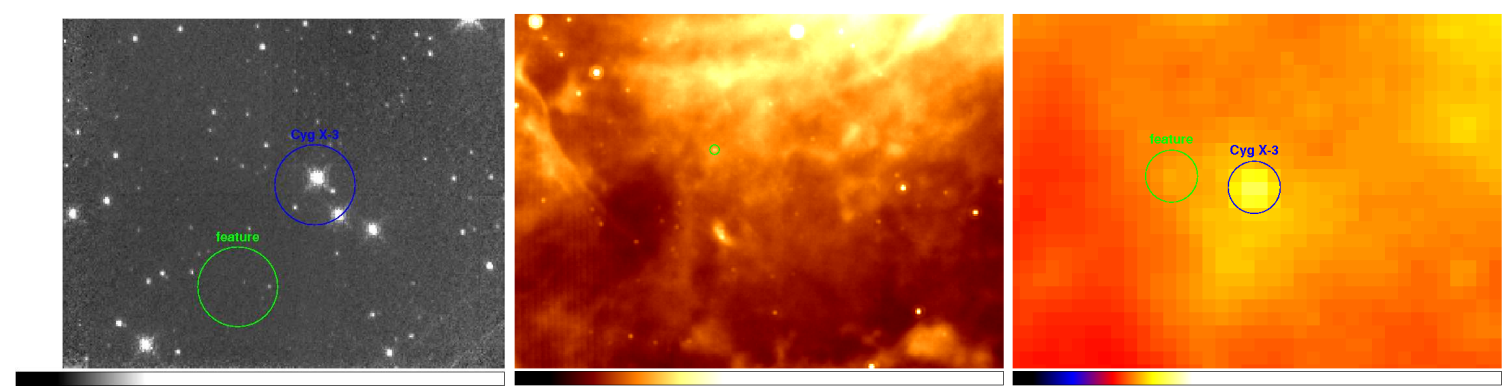

Figure 3: Left: A HST/NICMOS observation of the Cygnus X-3 region taken at 1.76 microns. Cygnus X-3 and the location of the feature are labeled. No obvious counterpart is seen. Center: Cygnus X-3 region at 24 microns (Spitzer/MIPS) with Cygnus X-3 labeled. Right: An enlargement of the region around Cygnus X-3. This shows indications that there might be extended IR emission associated with Cygnus X-3 and possibly the feature.

\section{Properties of the Feature}

\subsection{Temporal Variability of the Feature}

We first seek to examine the feature's temporal variability. The feature is located on a large scattering halo that is due in part to the telescope optics and in part due to dust along the path to Cygnus X-3. This scattering halo demonstrates the same $4.8 \mathrm{hr}$ orbital variation which is also observed in Cygnus X-3. Hence careful background subtraction is important in determining if the feature is undergoing any temporal variation.

In Fig. 4 are three light curves. The first is the feature without background subtraction. The second is the light curve from a background region selected to be the same angular distance from Cygnus X-3. Note that it shows the same orbital modulation seen in Cygnus X-3. Finally the third shows the background subtracted light curve for the feature. This final light curve appears to show a similar orbital modulation as seen in Cygnus X-3, but with a phase shift of 0.5 relative to Cygnus $\mathrm{X}-3$.

\subsection{Phase Relationship with Cygnus X-3}

To examine this possible phase relationship between the feature and Cygnus $\mathrm{X}-3$ the arrival time for each event was used to calculate a "Cygnus X-3" phase value. Then photons falling into certain phase ranges were broken in separate "phase" images. These images were assigned a certain color and combined to form a color coded phase image (see Fig. 5). The bands were: (red) $0.3-0.63$, (green) 0.63-0.96, and (blue) 0.96-0.3. Note the blue color of the feature. This indicates that bulk of the photons are arriving in the 0.96-0.3 phase range. It is also important to note that no background subtraction was done and hence there is no issue with the background subtraction creating a false time/phase variation of the feature.

One ramification of this is that one can create phase selected images in which the feature appears and vanishes as a function of phase (see Fig. 6). One can also see this more dramatically in the movie which accompanies this paper.

Finally in Fig. 7 is the phase folded light curves of the background and the feature. It can clearly be seen that the feature exhibits the same slow rise and rapid drop that one sees in Cygnus 

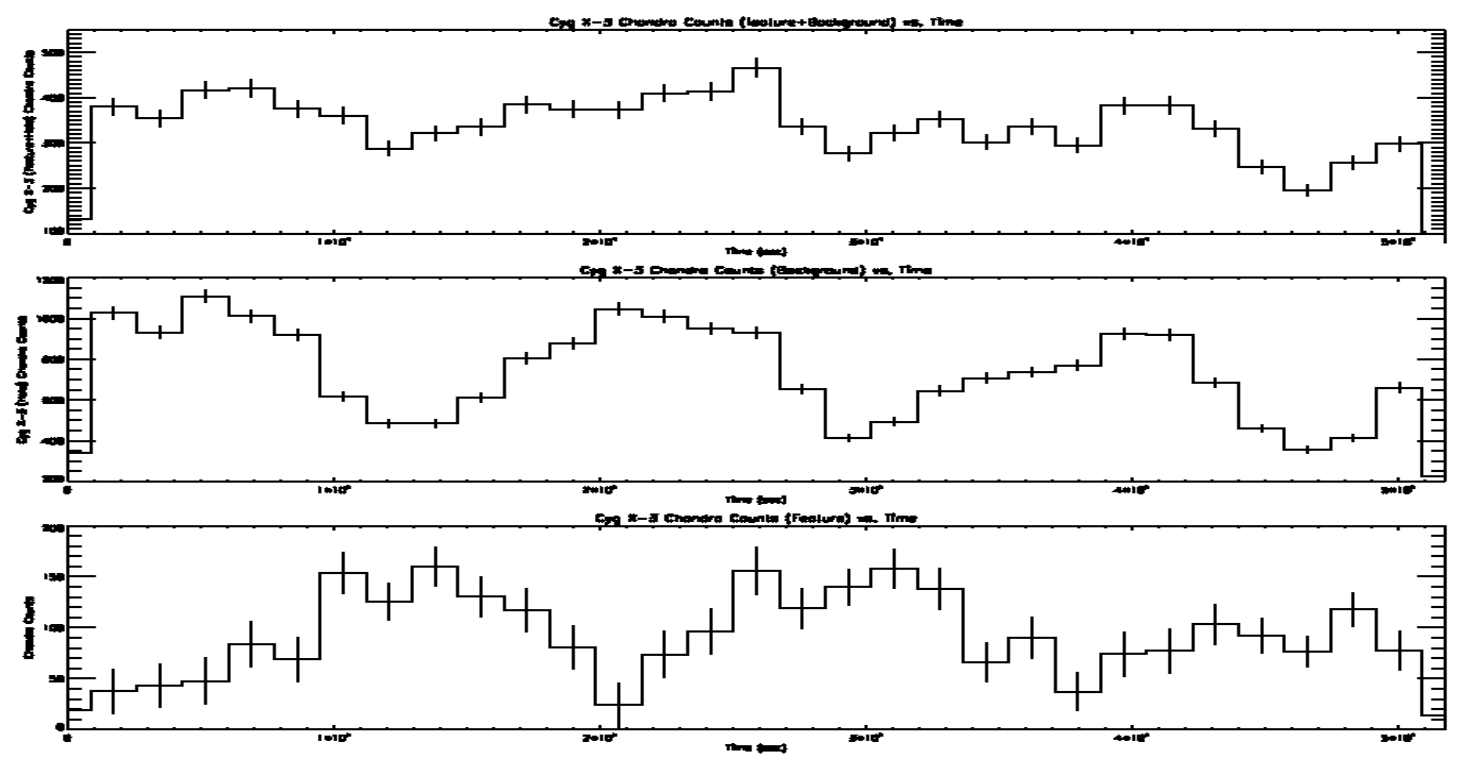

Figure 4: Top: Light curve of feature without background subtraction. Middle: Light curve of the background area showing the orbital modulation of Cygnus X-3. Bottom: The light curve of the background subtracted region of the feature.

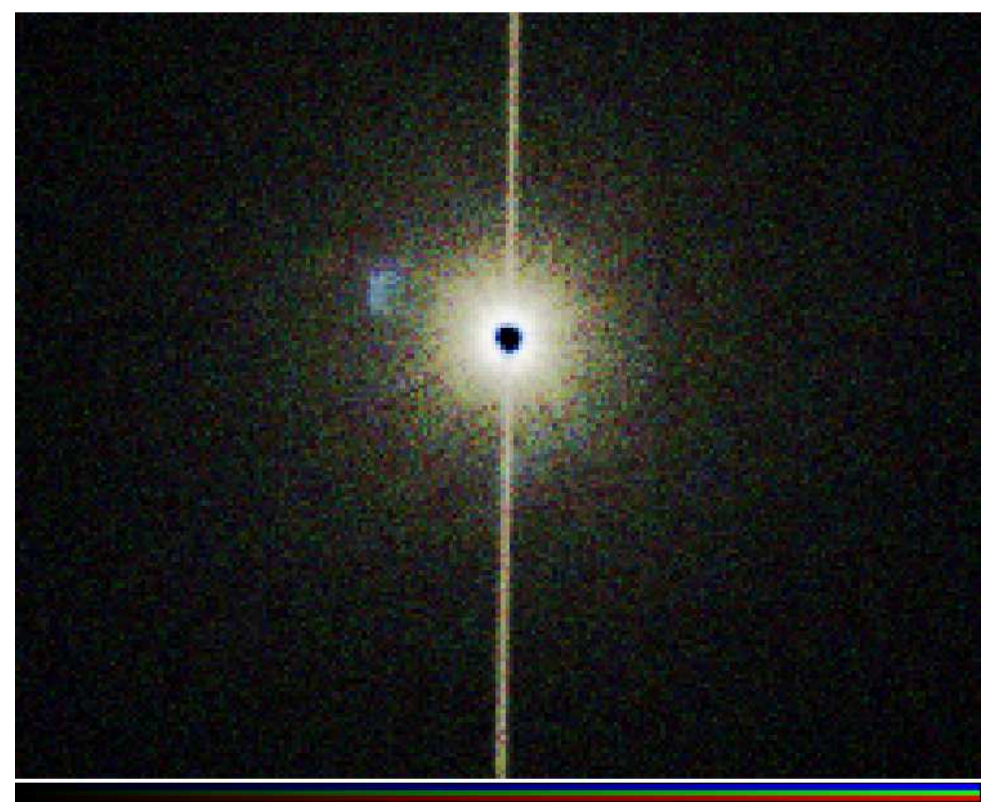

Figure 5: A color coded phase image of Cygnus X-3 area. Note the blue color of the feature which indicates that the bulk of the photons are arriving in the 0.96-0.3 phase range.

X-3 but with a phase lag. It is also noted that this would not be what one would see if this was due to a background subtraction issue. From centroid fits to the peaks of a cross correlation between the background and the feature a phase lag of $0.551 \pm 0.003$ is found. It should be noted that all of the Chandra data sets show an anticorrelation between the light curve of the feature and background.

\subsection{Flux Behavior}

To examine how the phase averaged flux of the feature changed between observations a photon 


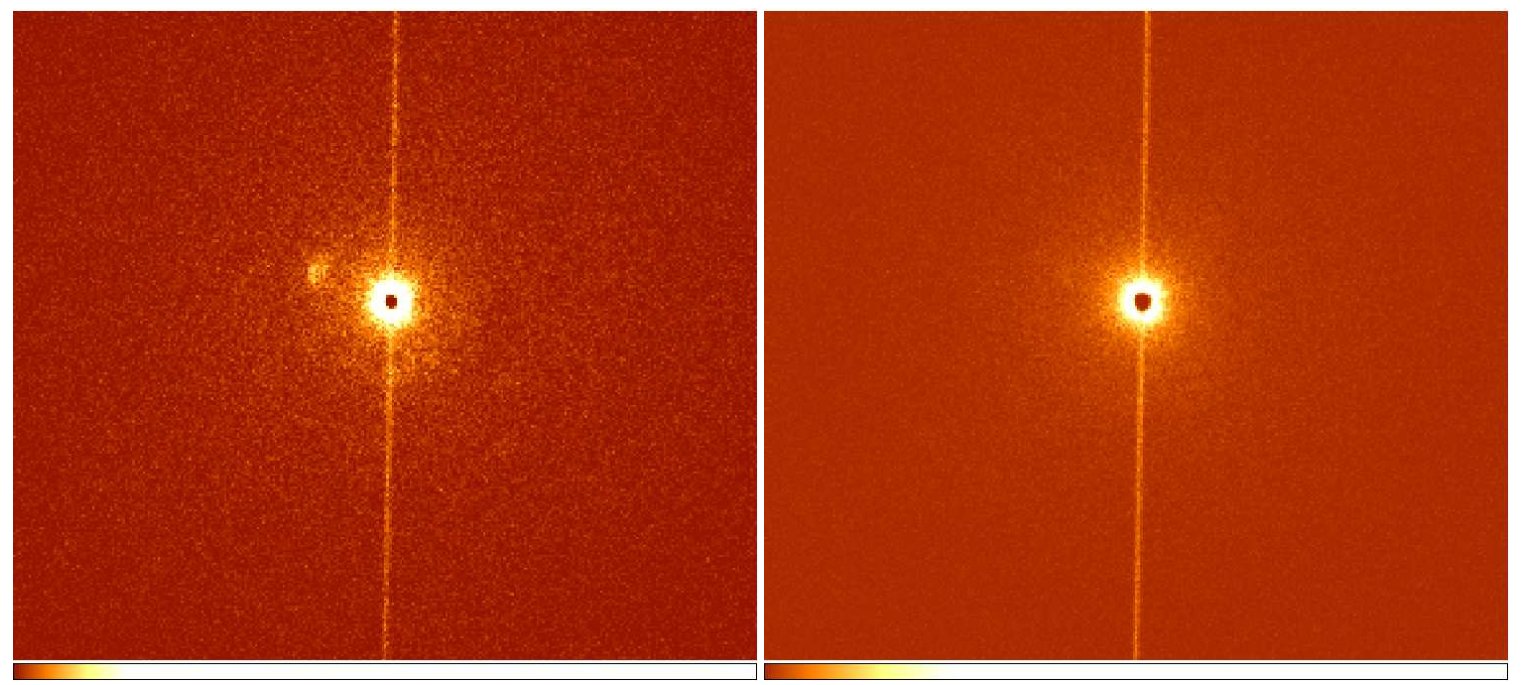

Figure 6: Phase selected images. left: Phase range 0.96-0.3 image. Now you see it. right: Phase range 0.5-0.8 image. Now you don't.
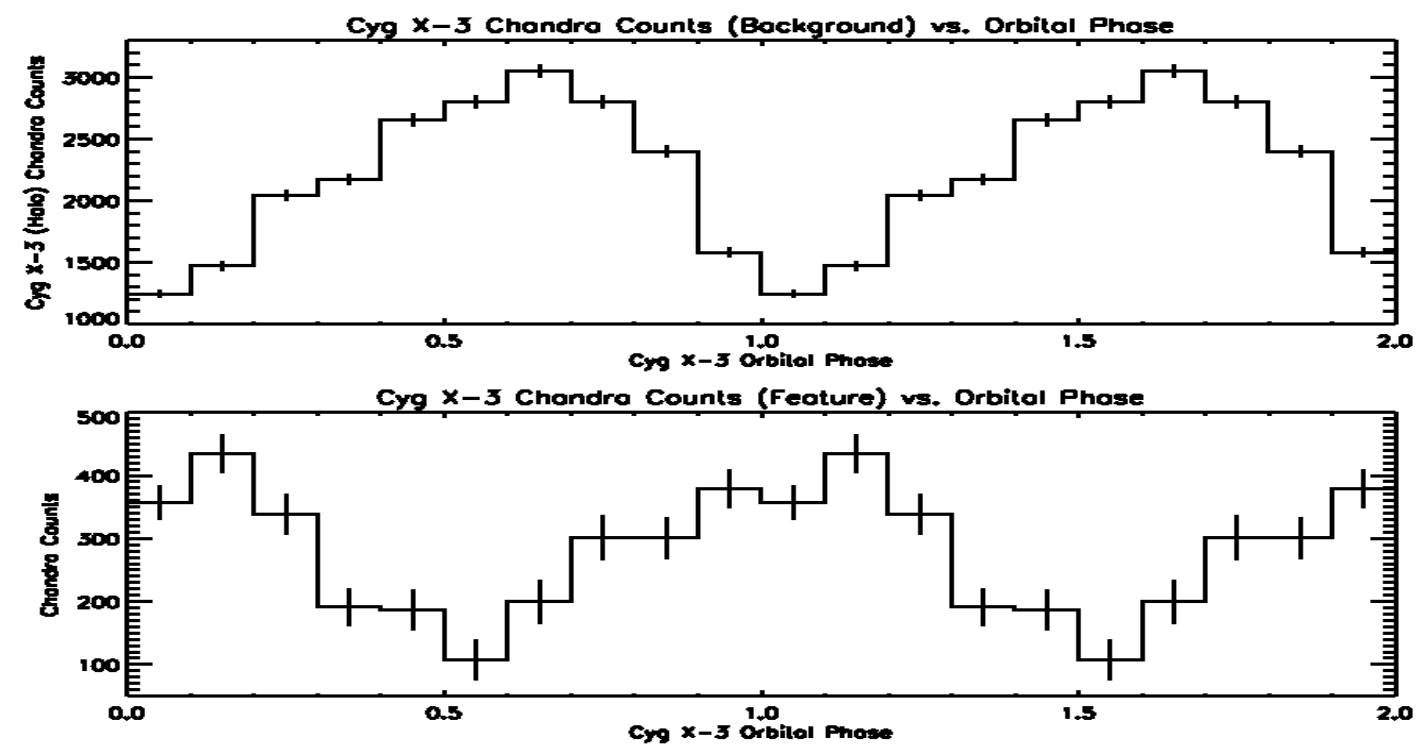

Figure 7: Folded light curves. Top: Phase folded light curve of the background region. Bottom: Phase folded light curve of the background feature. Note the similar of the light curves and the 0.5 phase shift.

flux of the feature was calculated for each of the Chandra/ACIS observations. This entailed creating an exposure map for each observation (using an energy of $3.5 \mathrm{keV}$ which is generally near the peak of the spectrum of the feature) and then dividing the image by the exposure map. Then by comparing the feature's region to that of the background a photon flux can be estimated for each data set. These values were in turn compared with RXTE/ASM count rate for Cygnus X-3 for each observation. A plot of this is given in Fig. 8. There appears to be a direct correlation between the flux of the feature and that of Cygnus X-3. 


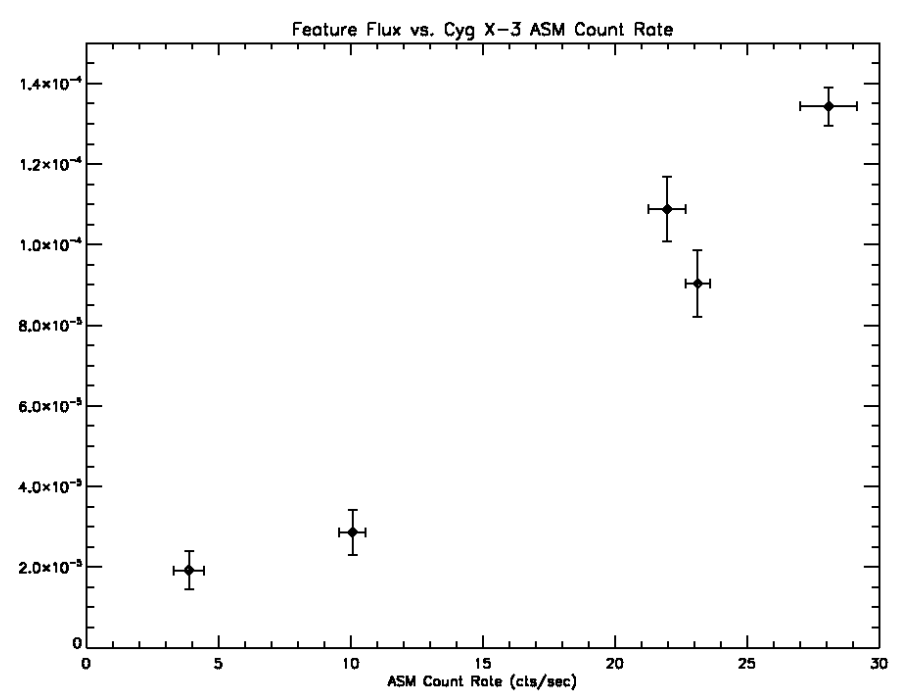

Figure 8: A plot of the photon flux of the feature vs. the RXTE/ASM count rate of Cygnus X-3 at the time of the observation. Note the apparent correlation.
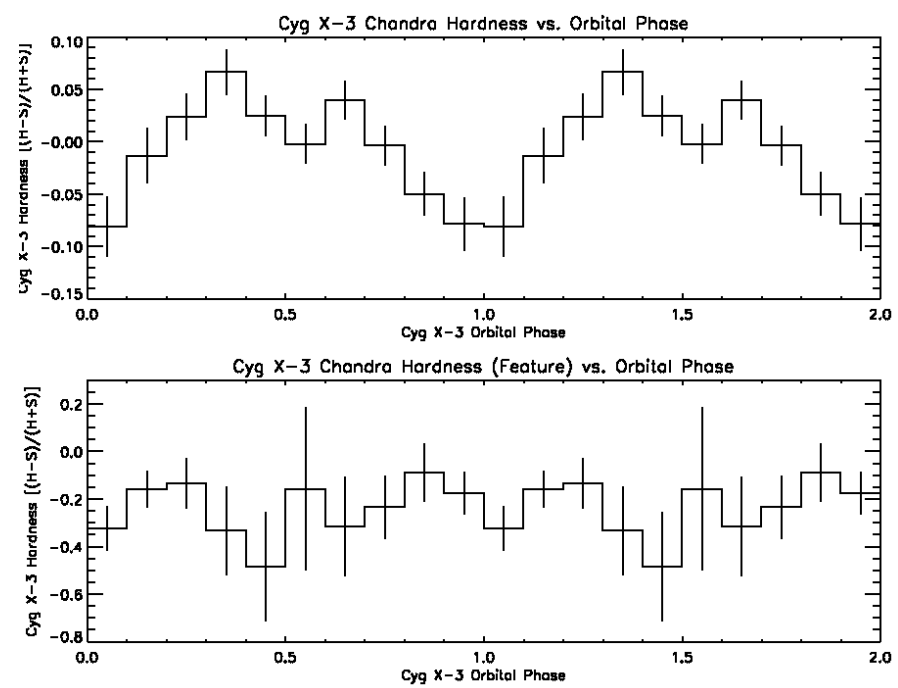

Figure 9: Hardness ratio as a function of orbital phase. Top: Hardness ratio of Cygnus X-3 (halo). Bottom: Hardness ratio of the feature. Note the lack of any orbital modulation.

\subsection{Hardness Ratio}

To better understand the nature of this feature we created a hardness ratio for both Cygnus X-3 (halo) and the feature. We used an energy range of 1-8 keV and divided the data into two bands. We chose a dividing energy of $4.33 \mathrm{keV}$ which gives an equal number of counts in both of the bands for Cygnus X-3. We also calculated the hardness ratio in a manner such that 1.0 means all of the counts are in the hard band and -1.0 means all of the counts are in the soft band. We find: (a) Cygnus X-3: $0.000 \pm 0.006$; and (b) Feature: $-0.223 \pm 0.041$. The feature is "softer" than Cygnus X-3. Also Cygnus X-3 shows orbital spectral variation and the feature does not (see Fig. 9).

\subsection{Spectrum}

In Fig. 10 are plots of Cygnus X-3's spectrum and the spectrum of the feature.

Cygnus X-3's X-ray spectrum shows a photoionized spectrum with: (a) lines of $\mathrm{H}$ and $\mathrm{He}-$ like heavy elements; (b) P Cygni profiles in most of the lines; and (c) strong Radiative Recom- 

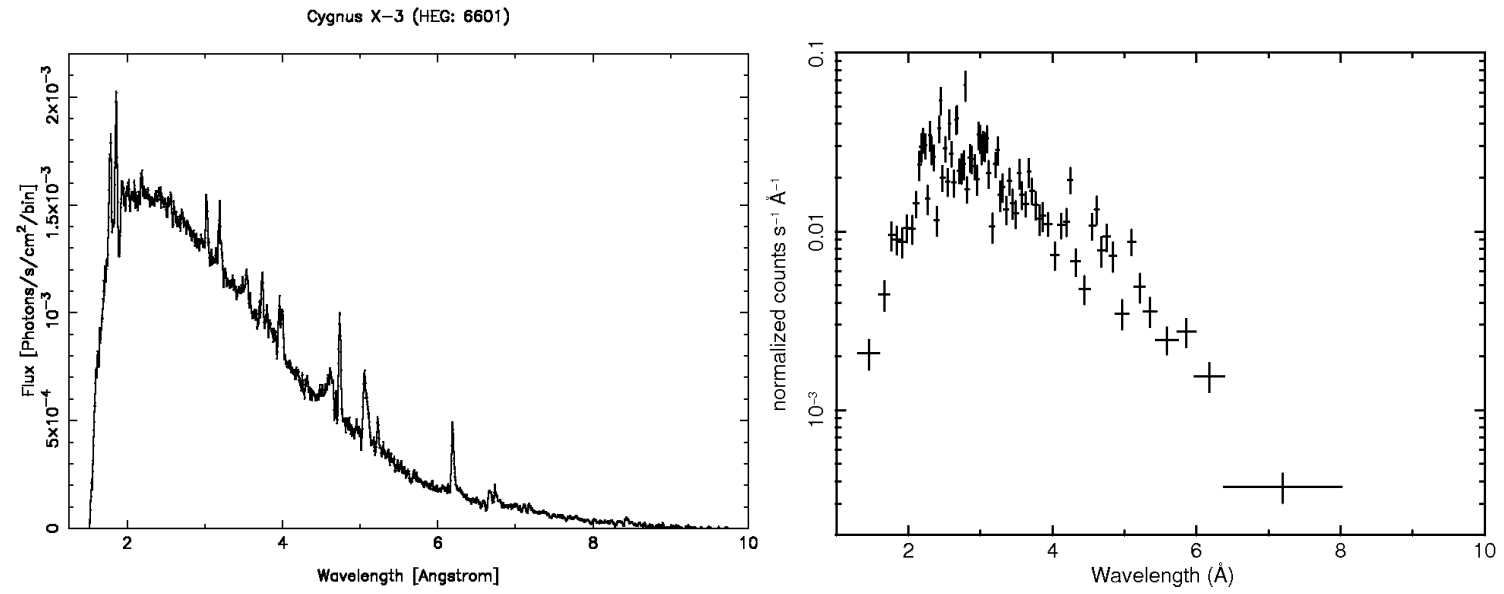

Figure 10: left: Chandra HETG grating spectrum of Cygnus X-3. Note the rich photoionized spectrum. right: $\mathrm{CCD}$ background subtracted spectrum of the feature. Note the lines (absorption and emission) and the reduced hard emission.

bination Continuum indicating plasma temperatures of between $30-50 \mathrm{eV}$.

The feature's spectrum is more problematic in part because of the issue of background subtraction. The background in this case contributes a much larger share of the photons than does the feature. So background subtraction is critical. An initial attempt to background subtract the spectrum are shown in Fig. 10. Fitting results show: (a) Simple continuum fits (power law, black body, etc.) give poor fits; (b) all fits need heavy absorption $\left(0.6-1.3 \times 10^{23} \mathrm{~cm}^{-2}\right)$; (c) there is little flux below $2.0 \mathrm{keV}(6 \AA)$; (d) line features (absorption and emission) appear present; and (e) there is missing hard flux when compared with Cygnus X-3.

\section{Possible Candidates}

What is Cygnus X-3's "little" friend? Any explanation most address the following: (a) the phase averaged flux of the feature is $\sim 10^{-4}$ of Cygnus X-3's flux and appears to be correlated with Cygnus X-3; (b) it has phase variations that appear anti-correlated with those of Cygnus X-3; (c) the time variation is $4.8 \mathrm{hrs}$ but the separation between the feature and Cygnus X-3 is $2.7 \mathrm{D}_{10}$ light-years if they are at the same distance; and (d) while the feature appears heavily absorbed it is also missing hard $\mathrm{X}$-ray flux.

\subsection{Instrumental Effect or Unrelated Source}

After careful examination of the data and discussion with the Chandra optics team we can find no optical/instrumental effect that would result in this feature. Due to the time/phase variations we rule out this being an unrelated source.

\subsection{Jet Impact Region}

It has been suggested that this might be a jet impact area [3].

Pro: With the strong wind from the Wolf-Rayet companion one might naturally expect a shell around the system with which the jets might impact and interact. 
Con: There are several issues which argue against the feature being a jet impact region: (a) The feature is not aligned with the radio jets. Why do we see the impact area with such a large offset? (b) How does one maintain the observed flux correlation? If the distance between Cygnus $\mathrm{X}-3$ and the feature is $2.7 \mathrm{D}_{10}$ light-years then how does one explain this flux correlation? (c) There is no obvious counterpart at other wavelengths. For a jet impact area one would expect there be emission at other wavelengths (IR in particular) since ISM/shell interactions would likely give rise to thermal processes. (d) Can one turn "on" and "off" the impact region fast enough? For an impact area one would normal expect a slowly fading residual flux. This does not appear to be the case. (e) Finally how easy is it to maintain a phase coherence with Cygnus X-3? This would have to represent a coherence train of pulses over the $2.7 \mathrm{D}_{10}$ light-year distance.

\subsection{Gravitational Lens}

The arc-like shape of the feature give some thoughts of gravitational lensing (Einstein's ring).

Pro: This could explain in a natural way the observed correlations and anti-correlations. It might be possible that strong lensing of the inner accretion disk near the compact object might give rise to the observed effects.

Con: But where is the lensing object located? This feature has been present at least since Chandra has been in orbit (over seven years). So any lensing object would have to have been in a stable location relative to Cygnus X-3 during this time. Also for Cygnus X-3's distance the mass of the lensing object would have to be very large. Based on an angular separation of $16^{\prime \prime}$ and weak gravitational lensing theory the mass would need to be of order $10^{12} M_{\odot}$ [5].

\subsection{Beamed X-Ray Emission}

Given the jets in Cygnus X-3 one might expect that beamed emission could provide a natural explanation for what is observed.

Pro: Could naturally explain the $4.8 \mathrm{hr}$ variation of the feature.

Con: There are several problems with beamed emission explaining the feature: (a) Generally one might expect beamed emission to be power law in form. This does not appear to be the case for the feature. Additionally the spectrum appears to contain absorption and emission lines. (b) There are no counterparts at other wavelengths. One would expect that beaming would also be seen at other wavelengths. (c) Why is it a anti-correlation? A correlation would seem to be more natural. (d) Why is the beamed emission at a much lower flux level than the normal X-ray emission from Cygnus X-3? (e) Finally there is the issue of misalignment between the X-ray feature and the observed radio jets.

\subsection{Scattering by a Cloud Along the Line of Sight}

Another possible explanation of this feature is X-ray scattering. Initial work on the Cygnus X-3 scattering halo give column density fits comparable to those found from fitting Cygnus X-3 X-ray data $\left(3-4 \times 10^{22} \mathrm{~cm}^{-2}\right)$ [6]. These fits indicated the presence of one of more clouds at $\sim 0.4$ of the distance to Cygnus X-3. This would correspond to most of the scattering (in the dust halo) occurring within the Perseus Arm of the galaxy. This feature could simply represent a dense cloud along our line of sight to Cygnus X-3. Features like this were expected to be observed with ROSAT. Various explanations were given for why they have not been seen [7]. 
Pro: This explanation would naturally lead to the correlations and anti-correlations which are observed. Where the phase difference is simply a difference in paths of the scattered photons. This could also explain the flux correlation and overall flux difference (factor of $\sim 10^{-4}$ ) since one would expect only a small fraction of the total flux to be scattered toward the observer. This could also give rise to a natural explanation of the spectral differences. The lose of the flux below $2 \mathrm{keV}$ is the result of additional absorption in the cloud and the reduced flux at higher energy simply reflects the drop in scattering efficiency at higher energies.

If we assume that this feature is due to an individual cloud the time delay will give the distance to the cloud [8]. The time delay is given by:

$$
\mathrm{t}=\frac{\Theta_{\mathrm{obs}}^{2}}{2 \mathrm{c}} \frac{\mathrm{Dx}}{1-\mathrm{x}}
$$

Where $\mathrm{D}$ is the distance to the source, $\Theta_{\mathrm{obs}}$ is observed angular distance from the source, $\mathrm{x}$ is the fractional distance of the scatter to source, and $\mathrm{c}$ is the speed of light. For $\mathrm{t}=2.63$ hours, $\mathrm{D}=$ $9 \mathrm{kpc}$, and $\Theta=16^{\prime \prime}$. Then $\mathrm{x}=0.77$. Thus the cloud is much closer to Cygnus $\mathrm{X}-3$ (within $2 \mathrm{kpc}$ ). Note that there is a factor of $\mathrm{n}$ (number of Cygnus X-3 orbital periods) degeneracy. Thus the $2 \mathrm{kpc}$ is a maximum distance from Cygnus X-3 and the feature could be much closer.

Con: While these features have been predicted this would represent the first observation of such a feature. Nothing like this has yet to be identified in any other Chandra observation of X-ray binaries. Also the fact that this feature has an extended size gives rise to some issues in modeling the data.

\section{Summary}

We have presented new and unexpected results from the analysis of a feature associated with the microquasar Cygnus X-3. This feature shows flux and phase correlations with Cygnus X-3 even though it is a relatively large angular distance (16") from Cygnus X-3.

This feature had been thought to be a jet impact area [3]. We have shown there are problems with this interpretation. We have examined other possibilities and the most promising of these is the scattering from a cloud relatively near Cygnus X-3. While this has long been predicted to occur this would represent the first such detection of such a phenomena. Although some issues still need to be addressed, this represents a new and exciting discovery.

\section{References}

[1] M. L. McCollough, et al., ApJ, 517, 951, (1999)

[2] A. Szostek, A. A. Zdziarski, \& M. L. McCollough, et al. ,MNRAS, 388, 1001, (2008)

[3] W. A. Heindl, et al., ApJ, 588, L97, (2003)

[4] J. R. Sánchez-Sutil, et al., $A \& A, 479$, 523, (2008)

[5] P. Schneider, J. Ehlers, E. E. Falco J. R., Gravitational Lenses, Springer, (1999)

[6] L. Valencici, Private Communication, (2007)

[7] P. Predehl \& S. Klose, $A \& A$, 306, 283, (1996)

[8] Trümpler and Schönfelder, $A \& A, \mathbf{2 5}, 445$, (1973) 